\title{
Protée
}

\section{Au supermarché. Libertés et contraintes dans le temple moderne de la consommation}

\section{Maria Pia Pozzato}

Volume 29, numéro 1, 2001

La société des objets. Problèmes d’interobjectivité

URI : https://id.erudit.org/iderudit/030616ar

DOI : https://doi.org/10.7202/030616ar

Aller au sommaire du numéro

Éditeur(s)

Département des arts et lettres - Université du Québec à Chicoutimi

ISSN

0300-3523 (imprimé)

1708-2307 (numérique)

Découvrir la revue

Citer cet article

Pozzato, M. P. (2001). Au supermarché. Libertés et contraintes dans le temple moderne de la consommation. Protée, 29(1), 57-63.

https://doi.org/10.7202/030616ar
Résumé de l'article

Les courses au supermarché sont un bon exemple pour vérifier comment les liens qui constituent l'intersubjectivité peuvent être parfois influencés par des formes déterminées d'« interobjectivité ". Là où l'influence du Destinateur se fait sentir, dans le sens injonctif (la pièce de monnaie pour avoir le chariot) ou incitatif (collection de points, don, rabais), les Sujets ont tendance à renouer des liens sociaux ; par contre, quand les marchandises sont proposées sans intermédiaire (self-service), et que seule leur syntaxe propre est chargée d'engendrer des programmes d'achat, on note alors le retour de simulacres d'autosuffisance du Sujet par rapport aux autres Sujets. 


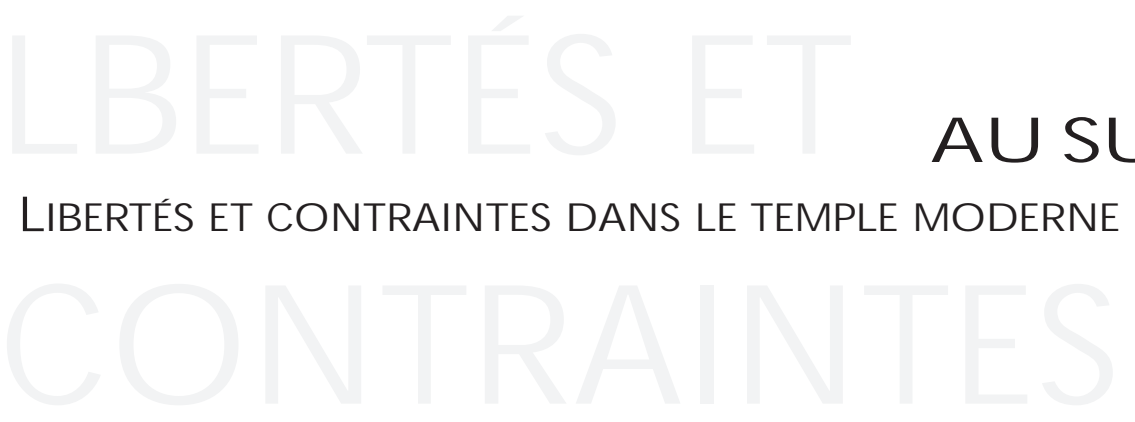

\section{AU SU PERM ARCHÉ}

Traduit de l'italien par Eric Landowski

MARIA PIA POZZATO

Depuis longtemps, l'ethnométhodologie et la sociosémiotique nous ont révélé

que sous nos comportements apparemment les plus banals se cachent des systèmes complexes de règles qui rendent significatifs même les plus petits détails du quotidien et qui confèrent à nos conduites une forte signification socioanthropologique. Les courses au supermarché en sont un bon exemple, comme cette étude se propose de le montrer: ce que j'entends en effet prouver, c'est que celui qui se rend au supermarché pour faire ses courses se soumet de son plein gré à un système intersubjectif et interobjectif extrêmement contraignant ${ }^{1}$.

La caractéristique essentielle du supermarché par rapport à une petite épicerie est l'absence de tout rapport direct entre acheteur et vendeur. D'où, en l'absence de négociation possible sur le moment - celui de l'achat-, la nécessité d'établir a priori les normes destinées à présider au processus d'achat, dans le but d'en assurer le meilleur résultat possible sous tous ses aspects.

\section{1. ÉPREUVE QUALIFIANTE ET ÉPREUVE GLORIFIANTE: LE CHARIOT}

Dans un supermarché italien moyen, le client affronte un premier système de normes au moment où il doit emprunter un chariot. Depuis quelques années, pour les achats les moins volumineux, on met à la disposition du client des paniers en plastique ou en métal. Ces paniers, qui seront redéposés en sortant, à la caisse, ne demandent aucune caution. En revanche, pour se procurer un chariot, le client doit insérer dans une petite boîte fixée près de la poignée, et par une fente prévue à cet effet, une pièce de cinq cent lires. Pour la récupérer une fois les courses finies, il faut replacer correctement le chariot dans l'espace aménagé à cette fin, où on trouve une sorte de tige permettant d'extraire la pièce de monnaie. Il est évident que, selon les catégories de la grammaire narrative classique, il s'agit là d'une épreuve qualifiante, grâce à laquelle le sujet acquiert le moyen de réaliser le "programme principal», c'est-à-dire les courses.

L'habitude tend à nous faire perdre de vue le caractère magique de cette transaction. Sur le plan mythique, la semi-disparition de la pièce de monnaie (seule une toute petite partie reste visible, comme pour garantir que notre avoir est toujours là) ressemble à un sortilège réalisé volontairement par le sujet. Récupérer la 
pièce sanctionnera la réalisation effective de l'épreuve glorifiante qui consiste à remettre le chariot à sa place.

Sur le plan pragmatique inhérent à l'échange économique, la dépossession momentanée et partielle de l'objet est au contraire un emprunt sur caution: le Destinateur-supermarché dit au client qu'il lui permet d'utiliser le chariot à condition de le remettre ensuite à sa place, sous peine, dans le cas contraire, de ne pas lui restituer son argent. Je peux dire, après une longue observation, que tout le monde, une fois les courses finies, accepte de faire un long parcours, depuis la voiture jusqu'à l'emplacement réservé au rangement des chariots, même sous une pluie battante, à seule fin de ne pas perdre ladite pièce de monnaie. Seule une configuration mythique est en mesure d'avoir une telle force de persuasion. Il me semble en effet peu probable que l'efficacité de ce contrat tienne à la somme d'argent en jeu, qui est vraiment dérisoire (cinq cent lires équivalent à un quart de dollar). Du reste, depuis tant d'années que ce dispositif est en vigueur, personne ne ressent le besoin d'augmenter la valeur de l'enjeu, en partie parce que cela exigerait des coûts importants pour la modernisation des chariots, en partie parce que les pièces de monnaie de mille lires en circulation sont encore trop rares, mais surtout parce que le système fonctionne très bien tel qu'il est, et cela même si les appareils se bloquent souvent, faisant du coup perdre du temps, et parfois la pièce. D'où un arrangement fréquent entre clients respectivement en train de commencer ou de terminer leurs courses, les premiers demandant expressément aux seconds de leur céder leur chariot contre la somme établie. Dans ce cas, le passage des cinq cent lires directement des mains d'un client à celles d'un autre crée une sorte de solidarité éphémère entre eux, à l'insu du Destinateur. Il ne faut pas sousestimer la valeur de cette pratique, car même si les pays dits latins ont la réputation d'être des oasis où tout le monde se sourit et se salue chaleureusement, en Italie il faut en réalité vaincre une certaine réticence pour s'adresser à un inconnu et lui demander une faveur, même aussi insignifiante. Ceci nous permet de mesurer combien l'insertion et le retrait de la pièce de monnaie sont fastidieux, mais combien ils sont aussi impératifs, au point de créer de nouvelles formes ad hoc de lien social.

Nous voyons donc qu'avant même de commencer les courses, nous sommes déjà entrés dans un système contractuel très fort, complexe et pas vraiment rassurant. Le programme d'usage «acquisition du chariot» crée simultanément deux simulacres: celui d'un Destinateur manipulateur et garant des valeurs, et celui d'un Sujet virtuellement en faute, en qui on ne peut avoir confiance. La nécessité de donner en gage la pièce de monnaie pour obtenir le chariot, l'obligation humiliante et un peu infantile de remettre les choses à leur place après les avoir utilisées placent la clientèle dans une situation de surveillance expresse. C'est comme si la direction du supermarché disait à chaque client: "Je te laisse entrer, mais sache qu'on est en train de s'assurer que tu ne violes pas les règles".

Multiplié par mille (car on trouve le même dispositif dans presque tous les supermarchés du pays), cela donne l'image implicite d'une société malhonnête qui aurait besoin de "pouvoirs forts" pour bien fonctionner. Celui qui accepte la règle, accepte en fait d'appartenir à une société de ce type. Et il n'est pas évident qu'un traitement aussi coercitif de la part d'un vendeur présent en personne serait accepté par l'acheteur. C'est l'impersonnalité qui le rend possible, et peut-être aussi le fait que la pièce n'est jamais tout à fait soustraite au client. Mais imaginons qu'à l'entrée d'un magasin, une vendeuse nous dise: «Donnez-moi cinq cent lires que je vous restituerai au moment de sortir, à condition que vous n'ayez pas sali la moquette». Personne ne donnerait la somme demandée, et chacun aurait "raisonnablement" une réaction d'indignation. Pourtant, les deux contrats sont en substance très similaires.

\section{SOUVERAINETÉ ET ACTUALISATION DANS LE PROGRAMME DES COURSES \\ En possession de sa demi-pièce de monnaie qui attend de redevenir entière, notre héros entre finalement dans le supermarché. Il laisse un triste}


parking à ciel ouvert, ou un souterrain encore plus sinistre et parfumé de gaz d'échappement, où on l'a traité comme un individu potentiellement antisocial, et le voici qui se trouve tout à coup dans un vaste espace resplendissant de lumière. Et surtout, le voilà, à partir de ce moment, qui commence à jouir d'un véritable régime de liberté: modalisé encore il y a peu par le seul /devoir faire/, il entre maintenant dans le royaume du / vouloir faire/ et du / pouvoir faire/ (et bien sûr, aussi, du / pouvoir ne pas faire/).

Investi de ce nouveau statut modal - celui de la souveraineté-, l'utilisateur moyen du supermarché adresse rarement la parole à qui que ce soit. Sauf exception (nous y reviendrons), aucun contact ne s'établit entre lui et les vendeurs, et les demandes d'aide qu'il peut adresser aux employés occupés à mettre de l'ordre dans les rayons ne reçoivent en général que des réponses laconiques, voire franchement bourrues. Le fait est que le client qui s'adresse au personnel bouleverse une des règles de base du supermarché, à vrai dire la raison même de son existence: le «self-service». Le personnel, qui est là pour d'autres raisons que pour servir la clientèle, ignore ostensiblement cette cohorte ininterrompue de gens en train de déambuler entre les rayons et d'étudier méticuleusement les marchandises, chacun pour son propre compte et à son propre rythme. Le programme des courses peut, en effet, être géré en totale autonomie: on pourrait, en théorie, rester huit heures dans un supermarché, lire les étiquettes de tous les produits, parcourir cent fois les mêmes couloirs, etc., sans enfreindre aucune règle. Les seules limites temporelles dépendent des heures d'ouverture et de fermeture du magasin, et la seule vraie interdiction est de consommer les produits. Si vous mangez ne serait-ce qu'un bonbon, cela peut occasionner une sanction, car la condition fondamentale de cette phase du programme - le maintien d'un statut actualisé mais non réalisé - serait alors violée. Tant que vous ne payez pas à la caisse, vous n'êtes pas en train d'acheter et donc vous ne devez pas consommer, même pas ce que vous avez déjà choisi et mis dans votre chariot, puisque votre choix demeure un choix révocable jusqu'au moment où vous aurez réellement payé.

\section{LE SUPERMARCHÉ} COMME UNIVERS ILLIMITÉ DE BIENS

Le statut des produits dans le chariot est donc très particulier. Ils font en un sens dejà partie de la sphère de possession du sujet qui les a désirés et choisis, et pourtant ils ne sont pas encore à proprement parler à lui. En termes sémiotiques, ils relèvent davantage du «vouloir avoir» que de l'«avoir», et à ce titre ils paraissent se situer paradoxalement à mi-chemin entre les objets de valeur modale et les objets de valeur descriptive. On sait que l'objet que nous désirons et que nous allons acheter est doté en général d'une «aura» - d'une «valence» - plus intense que l'objet que nous avons déjà acheté, ne fût-ce que quelques minutes auparavant. Avant la conjonction, l'objet ne fait pas encore partie de notre environnement extérieur et banalisé. Au contraire, son existence se situe encore tout entière à l'intérieur même de notre propre univers modal et passionnel - un peu comme, pour Swann, le visage d'Odette «pas encore embrassée».

Toutefois, les objets de valeur qu'on trouve dans un supermarché ont une prérogative particulière: si quelqu'un, par erreur ou par choix, s'approprie votre chariot ou les produits qu'il contient, aucun vrai conflit n'éclatera. Il ne saurait, en effet, y avoir de confrontation de ce type à l'intérieur d'un supermarché, étant donné que ce genre de commerce constitue le simulacre parfait d'un univers inépuisable de biens, où l'appropriation d'un objet de valeur par un sujet n'implique jamais la spoliation d'un autre sujet. Il y a donc une très faible valence d'appropriation dans les produits déposés dans le chariot et pas encore payés.

\section{LA DÉCISION D'ACHAT COMME PROCESSUS}

Du reste, le projet d'achat, en soi, est toujours in progress: personne n'entre dans un supermarché en sachant exactement ce que contiendra son panier à la sortie. Il existe certainement des structures 
programmatiques fortes, comme la «liste», mais elles sont en général corrigées et modifiées par des sollicitations contingentes. À côté des objets déjà déclarés manquants et qui figuraient donc d'avance «sur la liste», il y a les objets qui nous désirent, qui nous sollicitent, qui nous plongent dans des programmes décisionnels fugaces, mais non pas pour cela moins déterminants. Par exemple, l'objet inscrit sur la liste manque. Le subsituer par un autre plus ou moins équivalent, ou y renoncer? Ou bien: il y a un produit nouveau. L'essayer, ou non?

Les programmes suscités par les objets peuvent en outre être multiples et se trouver soit en conjonction, soit en disjonction polémique entre eux. Par exemple, ce qu'on appelle une «éco-recharge ${ }^{2}$ promeut deux programmes, avec deux valeurs de base différentes, qui, loin de s'opposer entre elles, travaillent en synergie pour inciter à acheter: une économie à réaliser, d'une part, et d'autre part une pollution plus réduite de l'environnement. D'autres produits créent au contraire un conflit chez l'acheteur potentiel. Par exemple, le "protecteur-lave-vaisselle» 3 prolonge peutêtre véritablement la durée de vie de la machine, comme le promet l'étiquette, mais il fait augmenter sensiblement le coût du lavage de la vaisselle. Sans parler du principal conflit qui se noue, pour tout acheteur, autour de ce qu'on appelle le « rapport qualité-prix".

Dans cette activité complexe de sanction à exercer sur les valeurs, où les désirs naissent, meurent, se confirment ou se transforment en quelques secondes, l'acheteur en puissance, s'il est seul, offre le spectacle d'une personne plongée dans ses propres réflexions, et s'il est en compagnie, celui d'un interlocuteur intensément engagé dans des échanges d'arguments relatifs au choix du menu du dîner ou du produit le plus avantageux. Mais en aucun cas les courses au supermarché, dans leur moment d'actualisation, ne mettent en jeu de liens contractuels très forts. On peut toujours changer d'idée et même, en ce cas, reposer le produit au hasard sans qu'aucun employé n'ose intervenir d'un: «Allez tout de suite reposer le parmesan là où vous l'avez trouvé!». Pourquoi autant de tolérance tout à coup? Parce que le moment du choix est conçu, délibérément, comme relevant du domaine d'une souveraineté de type infantile, où l'on joue avec les objets et où les objets se laissent posséder sans qu'il n'y ait rien à donner en échange, où les autres ne nous dérangent pas et ne sont pas en compétition avec nous, où personne ne nous presse, où les choses à acheter sont en nombre illimité, ou en tout cas si nombreuses que nous pourrions en théorie remplir dix, voire cent chariots. Et les lumières et la musique sanctionnent comme tel cet espace euphorique.

\section{LA GRAMMAIRE DES PRODUITS}

Je ne m'étendrai pas sur les relations syntagmatiques des produits dans un supermarché, déjà trop étudiées par les experts du marketing. On sait parfaitement que la disposition des différentes marchandises a une valeur persuasive non seulement pour faire acheter toutes sortes de choses, mais aussi pour orienter le client vers les marques qui rapporteront au vendeur le plus de bénéfice. Cette stratégie manipulatrice ne peut cependant transgresser une série de règles grammaticales qui imposent, par exemple, de ne pas juxtaposer les produits de nettoyage et les aliments. La différence entre ce qui est comestible et ce qui ne l'est pas (ou même pourrait être toxique en cas d'ingestion) reste fondamentale dans tout supermarché et ne peut pas même être ignorée dans ces «mariages interdits» entre objets que sont les offres spéciales où, en vertu du trait isotope "prix réduit», le paquet de café et le panettone de Noël par exemple se rejoignent, ou bien où, parmi les produits non comestibles, la poudre détersive pour carrelage se combine à celle pour laver la vaisselle. Ce désordre relatif des produits est censé, moyennant la réduction du prix, exercer un effet persuasif sur la clientèle. Il faut en effet supposer que l'affaiblissement des taxinomies induit alors le client à penser, par analogie, que les règles de l'échange économique se sont, elles aussi, relâchées: ce qui est présenté là en vrac, entassé et non plus rangé et ordonné, doit vraiment coûter moins cher, puisque cela échappe au 
système de classement habituel. C'est notre nature d'animaux profondément grammaticaux qui nous porte à penser de cette manière.

\section{VENTE Au DÉtAil ET RETOUR DE LA SENSORIALITÉ ET DE L'INTERSUBJECTIVITÉ}

L'économie n'est toutefois pas la seule valeur, bien au contraire. L'acheteur est soumis à des sollicitations esthétiques et esthésiques de divers types, comme le sait tout bon spécialiste du packaging des marchandises. De plus, depuis plusieurs années, les supermarchés italiens, même les plus grands, dits "hypermarchés», ont intégré dans le self-service une zone de vente au détail où, comme dans une charcuterie traditionnelle, on peut acheter au poids des aliments frais. C'est une zone hétérogène par rapport à l'espace soumis au régime solitaire et individualiste que nous avons décrit dans les paragraphes précédents. Ici, la dégustation, le parfum des produits alimentaires, la demande de conseils au vendeur, etc. réintroduisent l'intersubjectivité et la sensorialité dans le programme des courses. Mais ce faisant, on réintroduit aussi, entre clients, une conflictualité qui était complètement absente dans les espaces traditionnels du supermarché. Il faut faire la queue (habituellement avec billets numérotés), et si la personne qui vous précède achète beaucoup de choses, vous vous énervez. Il peut même arriver que le client d'avant vous soit parti avec le dernier morceau d'un produit frais en offre limitée.

En somme, avec l'option combinée self-service/vente au détail, tout se passe comme si se produisait une sorte d'oscillation entre des moyens et des fins incompatibles: grâce au self-service, on gagne en termes de rapidité, d'autonomie et d'absence de compétition, mais en contrepartie on perd l'avantage, justement, de la vente au détail, c'est-à-dire la possibilité de se faire donner des conseils et d'acheter des produits exactement au poids désiré, ou d'une fraîcheur particulière. Celui qui entre dans un supermarché sait qu'il devra se mesurer avec toutes ces différentes possibilités, et qu'il ne devra pas choisir seulement entre des marques et des produits mais aussi entre des régimes de temporalité et des types de contrats intersubjectifs. Pour cette raison, les achats au supermarché constituent un excellent exemple pour analyser comment les liens sociaux constitutifs de l'intersubjectivité peuvent parfois se former autour de formes d'«interobjectivité » bien déterminées.

\section{LA CAISSE, OU L'ACCÉLÉRATION DES ÉVÉNEMENTS}

Mais tel le foetus qui, après une période de béatitude inconsciente, doit se décider à abandonner le nid confortable qui l'a abrité pendant neuf mois, le client du supermarché, même s'il s'agit du plus relaxé et du plus flâneur, doit finir par se diriger tôt ou tard vers la caisse. Et là, comme le foetus justement, autant il avait été jusqu'alors câliné et laissé en paix, autant il se trouve soudain pressé, contraint, et finalement jeté, sans aucune possibilité de retour, dans un processus qui le dépasse.

Au moment où il fait la queue pour payer, le client entre, en effet, dans une chaîne qui le lie hiérarchiquement à celui qui le précède et à celui qui le suit. L'éventualité de changer un article, à la dernière minute, risquerait de rendre furieux tous les acteurs concernés, aussi bien les clients dans la file que les caissières. La tension du moment explique que le jeu devienne soudain aussi rude: aucun délai n'est plus permis, les produits, quand votre tour arrive, doivent être déposés rapidement sur le rouleau de la caisse et d'éventuelles décisions de dernière minute (par exemple relativement au nombre de sacs de plastique nécessaires) doivent être prises également très vite. Chaque «bip» émis par le lecteur magnétique des prix sanctionne la transformation du statut existentiel du produit, désormais uni par contrat avec la personne qui l'a choisi. Le fait de déposer des produits sur le tapis roulant constitue donc un acte tout à fait différent de celui consistant à les mettre dans le chariot. Mais le moment le plus dramatique est celui où, sans aucune considération pour la morphologie humaine, on doit en même temps, avec deux mains seulement et en quelques secondes, payer, prendre son reçu de caisse, ramasser sa monnaie, insérer trente-six objets de taille et de consistance 
hétéroclites dans deux ou trois sacs en plastique tout neufs, plats comme des soles, tout en s'assurant du coin de l'œil que personne n'emporte ce qu'on a déjà payé et reposé dans le chariot qui se trouve désormais juste au-delà de la barrière de la caisse. Pourquoi se dépêcher autant? Parce que vous y êtes contraint par les regards de la personne qui se trouve juste derrière vous, et par l'accumulation menaçante de ses achats tout près des vôtres, séparés par un minuscule diaphragme mobile qui risque d'écraser les oufs que vous venez d'acheter. Du point de vue modal et aspectuel, le paiement dans un supermarché est donc exactement à l'opposé du processus de choix des marchandises. Devant le tapis roulant, les stratégies subjectives se convertissent en tactiques et en automatismes, et il n'y a plus alors place ni pour les ralentissements ni pour les re-modalisations. Le sujet est purement et simplement devenu un engrenage.

Dès que le paiement a eu lieu, le contrat est pleinement réalisé et toute soustraction de produits par un tiers serait à partir de ce moment considérée comme un vol. La localisation et le régime de surveillance des produits changent donc: émietter par erreur des crackers pendant cette phase signifie emmener chez soi des miettes de crackers; ne pas surveiller son chariot après avoir passé la limite des caisses expose à d'amères surprises, alors que, comme on l'a vu, le vol, avant les caisses, n'avait pas de raison d'être puisque nous étions alors dans le monde de la non-possession. Ce sont là sans doute des choses évidentes, mais qui aident à comprendre le caractère catastrophique du changement de situation qui survient avec l'acte du paiement. L'ordonnancement des phases successives des courses au supermarché - chacune avec sa modalisation, sa pathémisation et sa tension propres - doit donc être relié à une stratégie manipulatoire précise, qui prévoit une injonction initiale (la pièce de monnaie dans le chariot), un «don modal» intermédiaire (la souveraineté dans le choix des produits), pour s'achever dans une phase fortement dysphorique, où le sujet se trouve littéralement forcé à transformer son statut d'acheteur encore seulement actualisé en celui d'acheteur enfin réalisé.

\section{LE SUPERMARCHÉ COMME GÉNÉRATEUR} D'IDENTITÉ COLLECTIVE: LE CLIENT «ASSOCIÉ»

Pour conclure, observons les mécanismes fortement manipulatoires de fidélisation et d'adhésion adoptés par différentes chaînes de supermarchés, par exemple, en Italie du nord, par les supermarchés et hypermarchés Coop, qui ont pour slogan: «La Coop sei tu, chi può darti di più? ” ${ }^{4}$. Cette grande coopérative promeut la transformation de l'acheteur en «associé» par le biais d'un versement, un tantum, d'environ cinquante mille lires. Ce type de client-associé a droit, en échange, à la restitution, une fois par an, d'environ 0,5\% de ce qu'il a dépensé dans les supermarchés de la chaine. L'associé peut également verser à la coopérative des sommes supérieures, selon son bon vouloir, et voir ses dividendes annuels augmenter proportionnellement.

Dans d'autres supermarchés, il existe des cartes de fidélité qui permettent aux seuls associés de bénéficier de «réductions» sur certains produits. La figure du client-associé est à l'opposé de la figure du sujet antisocial dont le Destinateur-supermarché doit se protéger. Mais peu importe qu'il y ait un syncrétisme de mise en scène parfait entre les deux rôles thématiques: pour emprunter un chariot, le client en possession d'une carte de fidélité doit insérer sa pièce de monnaie comme tout un chacun. Car c'est seulement d'un point de vue strictement économique que change la manière de le traiter.

Dans presque tous les supermarchés italiens, on peut par ailleurs trouver des «carnets de points» (en général, un point correspond à vingt mille lires de dépense) destinés à inciter le client à acheter plus régulièrement, en échange de cadeaux. Nous retrouvons donc la figure du Destinateur généreux qui, cette fois, offre des cadeaux hors de la logique strictement échangiste, presque en signe d'affection à l'égard du client fidèle. On pourrait croire qu'il s'agit là d'une forme très faible de persuasion, mais ce n'est pas le cas. Tout comme nous l'avons constaté à propos de l'extrême obéissance lorsqu'il s'agit de remettre les chariots à leur place, la collection de points peut aller aussi jusqu'à produire des aberrations, comme ces 
femmes ou ces hommes qu'on voit rester auprès des caisses pour se faire donner leurs points par des personnes qui, n'en faisant pas collection, sont prêtes à les jeter. Il est évident que cette forme postmoderne de mendicité n'a rien à voir avec la nature du cadeau en lui-même, puisqu'il s'agit en général de quatre assiettes ou d'une serviette de bain qui n'ont pas une grande valeur marchande. Le ressort est symbolique, c'est l'idée qu'il y a un cadeau et qu'à travers ce cadeau s'ouvre une échappatoire aux lois rigides de l'échange économique.

La stratégie manipulatoire et coercitive dans laquelle s'inscrit un programme aussi banal que celui des courses au supermarché ne peut que nous faire réfléchir sur les niveaux d'injonction autoritaire que la plupart d'entre nous sommes disposés à supporter en échange de sensations éphémères de souveraineté ou d'appartenance à d'inconsistantes identités collectives fondées, en l'occurrence, sur l'idée d'épargne et de bon rapport qualité-prix. Il est intéressant également de voir comment, là où l'influence du Destinateur se fait sentir, soit dans un sens injonctif (avec le chariot), soit dans un sens incitatif (avec la collection de points et les cadeaux), les Sujets tendent à renouer des liens sociaux, alors que là où les marchandises dominent sans intermédiaire, se manifeste comme une autosuffisance du Sujet par rapport aux autres Sujets. Il serait excessif d'en conclure que les comportements de consommation minent en général les rapports sociaux. Il n'en reste pas moins qu'au cour du concept de self-service ${ }^{5}$, il y a bien le simulacre d'un Destinateur "éloigné», presque camouflé, à l'ombre duquel le Sujet vit sa petite illusion individualiste de liberté.

\section{N O TES}

1. C'est à la réalité italienne que je me réfère en particulier, mais beaucoup de ces observations pourraient sans doute être généralisées à tout le monde occidental. Toutefois, il serait intéressant que cette réflexion donne lieu à une étude comparative avec les supermarchés de différents pays.

2. Sorte de sachet en plastique qui remplace le baril traditionnel en carton de poudre détersive pour machine à laver.

3. Produit dense, dégraissant, à utiliser de temps en temps pour le nettoyage intérieur du lave-vaisselle, assez coûteux en général. 4. «La Coop c'est toi, qui peut te donner davantage?». Cette grande chaîne, liée traditionnellement à des forces politiques de gauche, a beaucoup investi ces dernières années dans la publicité. Les annonces passées à la télévision pour la Coop par Woody Allen, par exemple, sont très célèbres.

5. Le discours pourrait donc s'élargir. De plus en plus, le self-service est utilisé aussi dans des domaines où était prévu, il y a encore peu de temps, la présence d'un opérateur, de la distribution de l'essence aux guichets de banque automatisés, ou à la restauration self-service.

\section{RÉFÉREN CES BIBLIO g RAPHIQ U ES}

BAUDRILlard, J. [1968]: Le Système des objets, Paris, Gallimard. CAPRETTINI, G.P. [1994]: «Aspetti metalinguistici nella comunicazione di marketing: sinonimia e decisione d'acquisto " (surtout § 2. Il modello supermercato nell'interazione prodotto-consumatore), dans $\mathrm{R}$. Grandi (sous la dir. de), Semiotica al marketing, Milano, Angeli. FLOCH, J.-M. [1987]: «La génération d'un espace commercial», Actes Sémiotiques, vol.IX;

[1989]: «La contribution d'une sémiotique structurale à la conception d'un hypermarché", Recherche et Application en Marketing, vol.IV, n².

Greimas, A.J. [1983]: Du sens II, Paris, Seuil.

Kehret-Ward, T. [1987]: «Combining Products in Use: How the Syntax of Product Use Affects Marketing Decisions », dans J. Umiker Sebeok (sous la dir. de), Marketing and Semiotics. New Directions in the Study of Signs for Sale, New York, Mouton-de Gruyter.

LANDOWSKI, E. [1989]: La Société réfléchie, Paris, Seuil, 1989; [1997]: Présences de l'autre, Paris, P.U.F.

LATOUR, B. [1996]: «On interobjectivity», Mind, Culture and Activity, vol. $3, \mathrm{n}^{\circ} 4,228-245$. 